Arthroskopie $2009 \cdot 22: 93$

DOI 10.1007/s00142-008-0487-6

Online publiziert: 29. März 2009

๑) Springer Medizin Verlag 2009

\author{
M. Galla ${ }^{1} \cdot$ P. Lobenhoffer ${ }^{2}$ \\ ${ }^{1}$ Ambulante Tagesklinik, Chirurgie im MEDICUM, Hildesheim \\ ${ }^{2}$ Klinik für Unfall- und Wiederherstellungschirurgie, \\ Diakoniekrankenhaus Henriettenstiftung Hannover gGmbH
}

\title{
Arthroskopische Therapiemöglichkeiten an Sprunggelenk und Fuß
}

teil, die Reposition der Gelenkfläche exakt beurteilen zu können.

Mit Beginn der 9oer Jahre wurden die arthroskopischen Techniken nicht mehr ausschließlich für die Behandlung intraartikulärer Läsionen angewendet. Das Arthroskop wurde nun am Sprunggelenk und Fuß auch zur Diagnostik und Therapie von Pathologien eingesetzt, die sich außerhalb des Gelenkkavums befinden. In den letzten Jahren erfreut sich die Methode der Rückfußarthroskopie zunehmender Beliebtheit. Extraartikuläre Strukturen wie beispielsweise ein symptomatisches Os trigonum oder eine Haglund-Exostose können mit der arthroskopischen Technik sehr gut dargestellt werden, ohne dass große Zugangswege erforderlich sind. Der entscheidende Vorteil dieser Vorgehensweise gegenüber offenchirurgischen Verfahren liegt in der geringen Morbidität und in den geringen Komplikationsraten bei gleich guten postoperativen Ergebnissen.

Die Ausdehnung des arthroskopischen Horizonts durch Einsatz des Arthroskops innerhalb einer Sehnenscheide wurde Ende der goer Jahre durch Prof. C.N. van Dijk in Amsterdam beschritten. Die von ihm propagierte Technik der"Tendoskopie" ermöglicht es, auch Pathologien der Sehnen an Sprunggelenk und Fuß minimalinvasiv zu adressieren.

In diesem Themenheft wird dem Leser die Einsatzvielfalt des Arthroskops am Sprunggelenk und Fuß näher gebracht. Neben der Vermittlung grundlegender arthroskopischer Operationstechniken am oberen Sprunggelenk sowie am Rückfuß werden die Behandlungsmöglichkeiten typischer intraartikulärer Krankheitsbilder des OSG wie das ventrale Impingementsyndrom und Knorpelläsionen des Talus dargestellt. Ein wichtiger Beitrag widmet sich der chronischen Instabilität des oberen Sprunggelenks, die oftmals mit intraartikulären Gelenkschäden assoziiert ist und bisher zu wenig Beachtung fanden.

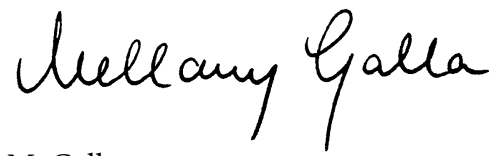

M. Galla

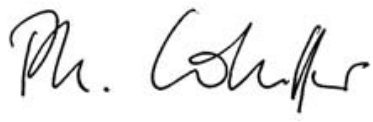

P. Lobenhoffer

\section{Korrespondenzadresse}

Dr. M. Galla

Ambulante Tagesklinik, Chirurgie im MEDICUM Goslarsche Landstraße 19, 31135 Hildesheim melgalla@web.de

Prof. Dr. P. Lobenhoffer

Klinik für Unfall- und Wiederherstellungschirurgie, Diakoniekrankenhaus Henriettenstiftung Hannover gGmbH Marienstr. 72-90, 30171 Hannover unfallchirurgie@henriettenstiftung.de

Interessenkonflikt. Die korrespondierenden Autoren geben an, dass kein Interessenkonflikt besteht. de bietet hier gegenüber der konventionellen Methode den unschlagbaren Vor- 